\title{
Birefringence Analysis of Directional Fiber Coupler Induced by Fusion and Coupling Parameters
}

\author{
${ }^{* 1}$ Dedi Irawan, ${ }^{1,2}$ Saktioto, ${ }^{2}$ Iwantono, ${ }^{2}$ Defrianto, and ${ }^{1}$ Jalil Ali \\ ${ }^{*}$ Advanced Photonic Science Institute, Faculty of Science, Universiti Teknologi Malaysia, Johor, Malaysia 81310 \\ Tel: +601116417916; E-mail: dedi.dawan@yahoo.com \\ ${ }^{2}$ Physics Dept, Math and Sciences Faculty, University of Riau, Pekanbaru, Indonesia
}

\begin{abstract}
The effect of fusion and coupling parameters to the form of birefringence of coupling region directional fiber coupler is reported. Vector of polarization was determined by using perturbation method that describes the propagation of electric field in single mode fiber coupling induced by stress which is caused by fusion and coupling parameters during fabrication. It is found that the radius of coupling cross section, refractive index different, normalized frequency, and twisting angle significantly affect the polarization and birefringence. But, the change of temperature during fusion in range of $27-1350{ }^{\circ} \mathrm{C}$ gives small increment in birefringence directional fiber coupler. By knowing these parameters, the polarization and birefringence are possible to maintain and optimize the fiber coupler.
\end{abstract}

Key words: Directional fiber coupler, polarization, birefringence, fusion and coupling parameters

\section{Introduction}

A directional fiber coupler is a key element in optical communication. It is used to split, combine, filter, and to switch an optical signal. Due to wide range application, this field becomes still important to be studied. The directional fiber coupler is fabricated by heating and pulling the coupling region until the pre-set coupling ratio is reached. Since the optical communication system requires devices with low lost and higher efficiency, the polarization may take more intention to be understood. The polarization is one of the main sources of pulse distortion that contributes to the system bit-error rate [1]. It is significantly caused by the fusion and change of coupling parameters during fabrication directional fiber couplers.

In conventional super mode analysis, the electric fields are constructed by symmetric and anti-symmetric superposition of unperturbed fields of each fiber in isolation from other fibers. This leads to obtain propagation constants of super mode by perturbation technique or variation methods [1-2]. The polarization was analyzed based on the distance different of coupling power. The modified Bessel function was used to describe the behavior of decreasing cladding field terms with azimuthal order [3].

The polarization characteristics of directional fiber coupler also were studied by *Corresponding author: Dedi Irawan Address: Advanced Photonic Science Institute, Faculty of Science, Universiti Teknologi Malaysia, Johor, Malaysia 81310. E-mail address: dedi.dawan@yahoo.com, Phone: +601116417916 
[4]. It investigated how the output power varies by applying State of Polarization (SOP) to the input power. The modeling was focused to the degree of fusion using Mueller matrix method. It was found that the Polarization Dependent Loss (PDL) much increases as the degree of fusion is weaker [4-5]. In recent years, the dependent of wavelength to the polarization in directional fiber coupler was also studied [6]. It stated that the wavelength dependences increase if the fibers become closer. At the same time, it was found that the light signal more polarized to $\mathrm{x}$-direction than $\mathrm{y}$-direction caused by elliptical geometry of fiber's core. However, for weakly coupling which means the fibers just touching only, the wavelength and the polarization dependences increase significantly if the distance between the fibers' core is longer [7].

In this study, the analysis of polarization and form of birefringence are focused on the fusion region. The vector of polarization is determined as function of wavelength, refractive index different, and temperature. This effect leads to change the form of birefringence at coupling region. By knowing these parameters effects gives improvement of and better performance fiber coupling.

\section{Birefringence of Fiber Coupler Induced by Coupling Parameters}

The interaction of electric field $\mathbf{E}$ in dielectric media, fiber optics, is always polarized in to $x, y$ direction. It significantly depends on the permeability of the medium interaction $\varepsilon$. This can be mathematically expressed as follows.

$$
\mathbf{P}=\Delta \varepsilon \mathbf{E}
$$

Since the permeability of dielectric medium is described by the refractive index profile of its material, it can be written,

$$
\varepsilon=\varepsilon_{0}\left(n^{2}\right)
$$

Deformation of coupling region during heating and pulling process leads the polarization phenomenon in fiber coupler. An optical signal passes through and split at the junction much affected by the geometrical coupling region. As in previous description, It has been shown the cross section varies as in form of taper and it is not perfectly symmetry anymore. This external stress causes light wave polarized to $\mathrm{x}$ and $\mathrm{y}$ direction. In other hand, the electric field propagates in fibers is broken becoming two unidentical component that travel with different velocity. This property is called birefringence.

The birefringence and polarization mode dispersion (PMD) as function of temperature shows that the birefringence increases exponentially versus $V$-parameters. It may be useful to determine form of birefringence if the fibers are joined as fiber coupler. It also was studied on the birefringence of two identical step index cores based on weakly coupling. Form birefringence is analyzed using perturbation method. From point of view, it is focused on the strength of coupling to $\mathrm{x}$ and $\mathrm{y}$ polarized component, so that this method is no longer use for analyzing form birefringence induced by thermal and geometrical stress.

In this study the birefringence is analyzed due to internal and external stress such as wavelength, temperature, geometry, and pressure. Basically, the fabrication of 
directional fiber coupler based on fusion and elongation methods operates with twisting, then heating the coupling region until $\approx 1350$ ${ }^{\circ} \mathrm{C}$ by injecting Hydrogen gas at pressure 1 Bar. Now, let write the general form of birefringence as follows. $B(\bar{r}, \lambda, T, P)=\left[\left(\frac{\partial B_{r}}{\partial r}+\frac{\partial B_{t w i s t}}{\partial r}+\frac{\partial B_{b e n d}}{\partial r}\right)+\left(\frac{\partial B_{T}}{\partial T}\right)+\left(\frac{\left.\partial B_{j}\right)}{\partial P}\right)\right]_{\lambda}$ be written as follows.
$D_{\text {coupling }}$
Where $\frac{\partial B_{r}}{\partial r}$ is the partial birefringence due to

Where $\frac{\partial B_{r}}{\partial r}$ is the partial birefringence due to geometrical changed. $\frac{\partial B_{t w i s t}}{\partial r}$ and $\frac{\partial B_{\text {bending }}}{\partial r}$ is partial birefringence due to twisting and bending effect respectively. $\frac{\partial B_{T}}{\partial T}$ and $\frac{\partial B_{P}}{\partial P}$ are partial of birefringence due to thermal and pressure effects.

Firstly, let solve the geometrical contribution the form of birefringence of directional fiber coupler. Since the cross section of coupling region is varied exponentially, and has the smallest cross section at the center, and the initial coupling region diameters is $2 r_{0}$, the diameter profile of tapered region can be expressed as follows.

$$
D_{\text {coupling }}^{\text {region }}=2 r_{0} \exp (l / \Delta l)
$$

Where $l$ is coupling length, and $\Delta l$ is heating zone. Since light is polarized to $x$ and $y$ direction, the birefringence due to geometrical change can be expressed as difference of the coupling component.

$$
\frac{\partial B_{r}}{\partial r}=r_{\substack{\text { coupling } \\ \text { region }}} \frac{V^{2}}{\Delta^{3 / 2}}\left(\kappa_{x}-\kappa_{y}\right)
$$

Where $V$ is normalized frequency, and $\Delta$ is percentage of refractive index different. The different of coupling coefficients due to stress in to $x$ and $y$ direction is

$$
\left(\kappa_{x}-\kappa_{y}\right)=\frac{2^{5 / 2}\left(n_{c o 2}^{2}-n_{c}^{2}(T)\right)^{1 / 2} U^{2}}{n_{c o 2}^{3 / 2} a \sqrt{\pi} V^{7 / 2}} \text { [7]. Вy }
$$

using this relation, and using Eq. (4) for

It can be simplified yields

Where $U=2.405$, and $a$ is fibers diameter at critical coupling region. Fig. 1 shows the linear increment of birefringence at varies radius of coupling cross section. At refractive index different $n$-diff $=0.26$, by varying radius in range of $2-4$ micron yields change in birefringence about $0.9 \times 10^{-}$

$$
\frac{\partial B_{r}}{\partial r}=\frac{D_{\text {coupling }}^{r \text { regin }}}{\Delta^{3 / 2} V^{3 / 2}} \frac{2^{3 / 2}\left(n_{c o 2}^{2}-n_{c}^{2}(T)\right)^{1 / 2} U^{2}}{a n_{c o 2}^{3 / 2} \sqrt{\pi}}
$$
6/ $\mu \mathrm{m}$. 


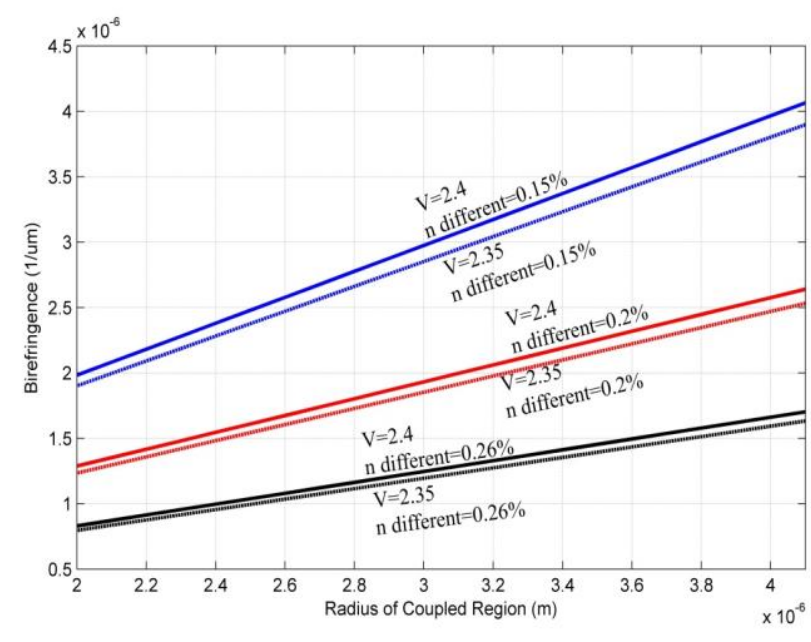

Figure 1. Change in birefringence due to change in

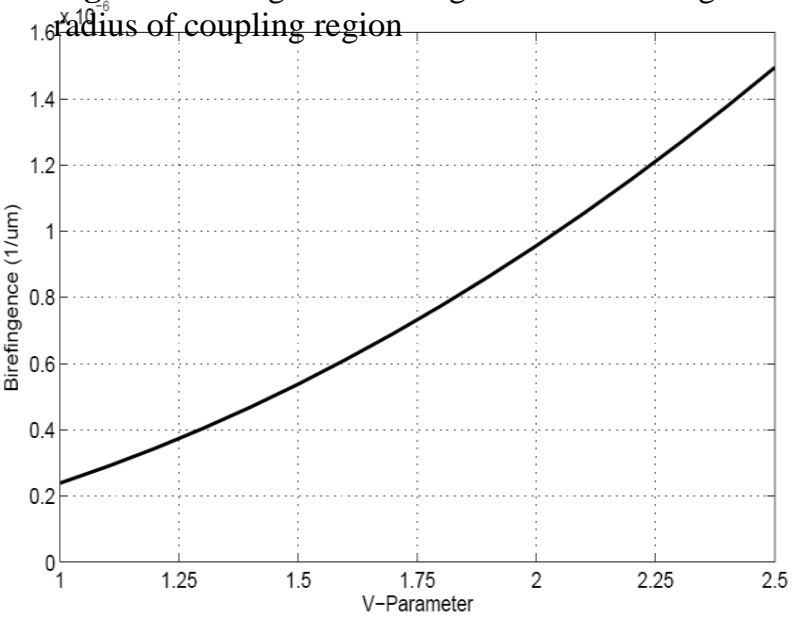

Based on Eq. (7), it can be seen that birefringence is affected by normalized frequency, or $V$-number. The variation of coupling radius gives more significant change in birefringence at lower $n$-different with higher $V$-number as shown by Fig. 1 . The higher degree of fusion causes the fiber's core and cladding reaches to make a compound. The more uniform the coupling region, the small different between the refractive index. Fig. 2 shows that the birefringence is exponentially increases with increasing the normalized frequency which is function of wavelength. It depicts how the fusion affects the core's radius and refractive index so that $V$-number is changes. As given by Eq. (7), modeling of temperature effect to the birefringence shown by Fig. 3 shows that there small change in birefringence in long range of fusion temperature. It is reasonable since the temperature of fusion does not change the properties of silica fiber. For $n$ different $=0.26$, increasing the temperature from $27-1350{ }^{0} \mathrm{C}$, changes the birefringence from 7.42 to $7.502 \times 10^{6} / \mu \mathrm{m}$. It can also be seen at constant temperature,

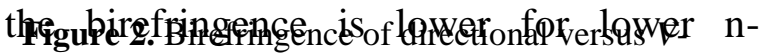
diffferfenters alue.

Beside the geometrical change due to heating and elongation effect, the stress of birefringence is also caused by bending and twisting. But, in this case, fiber coupler is placed on the pulling stages with controlled parameters, so that bending along the coupling region can be ignored. However, by twisting the coupling region, which is done before fusion, may take place in the calculation. The birefringence is proportional with the radius of coupling region, angle of twisting rotation, $\theta_{t r}$ and torsion $\varphi_{t}$. It can be expressed mathematically,

$$
\frac{\partial B_{t w i s t}}{\partial r}=\theta_{t r} \varphi_{t} r
$$

By knowing the ratio of $\theta_{t r} / \varphi_{t}$ such as given by reference $\theta_{t r} / \varphi_{t}=0.074$ [8]. Now Eq. (8) can be rearranged as follows.

$$
\frac{\partial B_{t w i s t}}{\partial r}=\left(\frac{\theta_{t r}}{\varphi_{t}}\right) \varphi_{t} r
$$




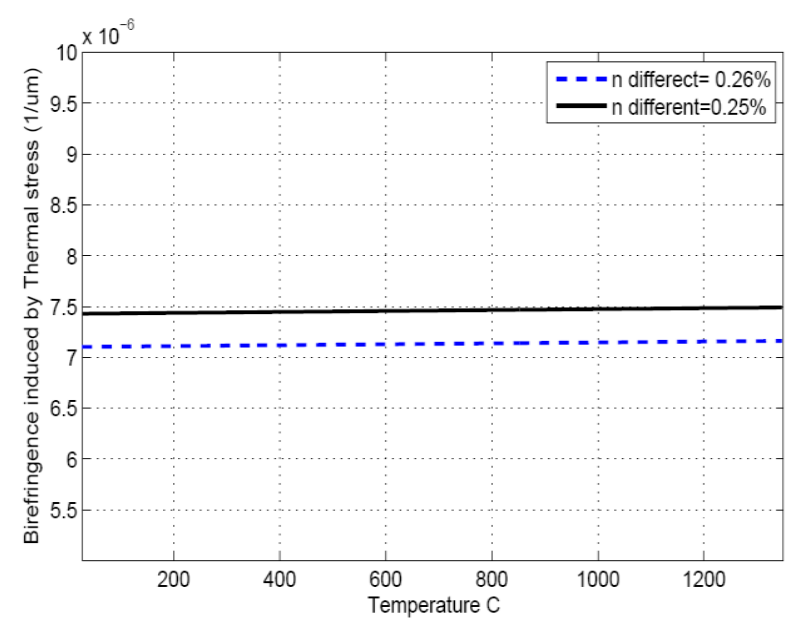

Figure 3. Effect of temperature to the change of

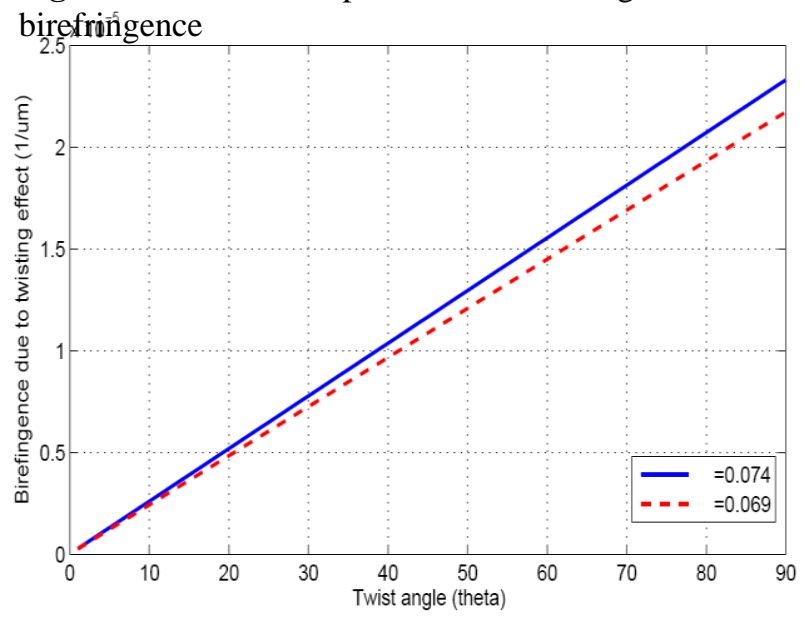

Fig. 4 shows the linear relationship between the changes of birefringence with twisting angle. It can be seen that by increasing the twisting angle very significantly affect the birefringence. Twisting effect contributes high birefringence in order $10^{5} / \mu \mathrm{m}$. It can be also seen that for higher ratio of $\theta_{t r} / \varphi_{t}$, the birefringence more increases as depicted by blue line in Fig. 4 .

Let us move on to the pressure effect to the form of birefringence. Birefringence occurs when there is external force such as pressure disturbing transversely the coupling region. If pressure $P$ is applied to the coupled region having radius $r$, the form of birefringence at certain wavelength can be written as follow [9-10].

$$
\frac{\partial B_{P}}{\partial P}=8 \frac{C_{p}}{\lambda}\left(\frac{P}{r}\right)
$$

Where $C_{P}=1195 \mathrm{~J} / \mathrm{Kg} . \mathrm{K}$ is specific heat [10]. Since the fabrication of directional fiber coupler is carried out in atmospheric pressure, it could not change the form of

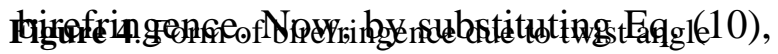
(9), and (7), in to Eq. (1), the birefringence characteristics of coupled region fused directional fiber coupler can be summarized as follow.

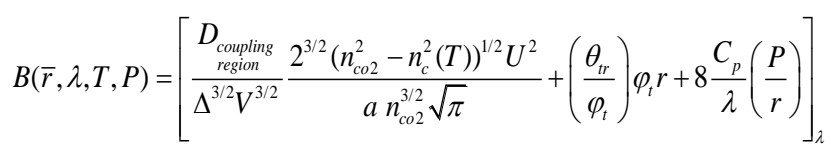

From the description above, it can be concluded that the form of birefringence is affected by the coupling and fusion parameter function. It have shown that the twisting angle of coupling region give significant change in birefringence compare with the radius of coupling cross section and the fusion temperature.

\section{Conclusion}

Birefringence analysis at coupling region very much depends on the geometrical change by fusion and elongation process. It is found that a significant change of birefringence contributed by the twisting angle, refractive index different, and the radius of coupling cross section. But, temperature of fusion only gives small changes in birefringence. However, power transmission of directional fiber coupler is 
exponentially decreases with increasing the angle of polarization vector.

\section{Acknowledgement}

We would like to thank the Institute of Advanced Photonic Science, Faculty of Science, Universiti Teknologi Malaysia (UTM) Sponsored by GUP Tier 1 No.QJ13000, 712601H02.

\section{References}

[1] T.L. Wu and H.C. Chang, "Rigorous analysis of form birefringence of fused fibre couplers", Journal of Lightwave Technology, Volume: 13, Page(s): 687 -691, (1995).

[2] Khare R. P, Fiber Optics and Optoelectronics, Oxford University Press. India (2002)

[3] Mary L. Boas, Mathematical Methods in the Physical Sciences (2005).

[4] S. Fu, P Shum., L. Zhang, W. Chongqing; A.M. Liu, Design of SOA-based dual-loop optical buffer with a $3 \times 3$ collinear coupler: guideline and optimizations, Journal of Lightwave Technology, Volume: 24, Issue: 7 On Page(s): 2768 - 2778 (2006)

[5] Z. Yabin, S. Xintong, Z. and Chao, "The Degree of Polarization-InducedFading for Fiber-Optic Sensor Based on $3 \times 3 \quad$ Coupler", Conference: Symposium on Photonics and Optoelectronics - SOPO , pp. 1-2, DOI: 10.1109/SOPO.2011.5780664, (2011)
[6] Chin-Lin Chen, "Foundations For Guided-Wave Optics, Perdue University, West Lafayette, Indiana, (2007)

[7] Snyder, A.W and Love. J. D, Optical Waveguide Theory, Institute of Advanced Studies Australian National University, Canberra, Australia 1983

[8] G. Ghosh, M. Endo, and T. Iwasaki, Temperature-Dependent Sellmeier Coefficients and Chromatic Dispersions for Some Optical Fiber Glasses, journal of lightwave technology, vol. 12, no. 8, august (1994)

[9] Saktioto, Jalil Ali, Mohamed Fadhali, Theoretical and empirical comparison of coupling coefficient and refractive index estimation for coupled waveguide fiber, Journal of Aplied Science and Engineering Technology. (2008)

[10] Wang, Y., Xue, D., Lu, X., Power transfer characteristics among $\mathrm{N}$ parallel single-mode optical fibers, Science Direct. Zhejiang University (2007). 